\title{
Clinical characteristics of pain originating from intra-articular structures of the knee joint in patients with medial knee osteoarthritis
}

\author{
Masahiko Ikeuchi, Masashi Izumi, Koji Aso, Natsuki Sugimura and Toshikazu Tani
}

\begin{abstract}
Purpose: Although disease progression of osteoarthritis has been well documented, pain pathophysiology is largely unknown. This study was designed with two purposes: 1) to characterize patients with knee pain predominantly originating from intra-articular structures and 2) to describe the location and pattern of their pain.

Materials and methods: 103 patients with medial knee osteoarthritis underwent an intra-articular injection of local anesthetics (joint block). At least 70\% pain relief was defined as positive for the joint block, while less than 50\% as negative. Pain characteristics in patients positive for joint block were evaluated in detail using a knee pain map.

Results: Sixty three knees (61\%) were positive and 33 knees (32\%) were negative. Patients negative for the joint block were significantly higher age, suffered for longer time, and complained more diffuse pain. Although pain at anterior medial area during walk was the most common finding, pain characteristics differed among different knee areas.

Conclusion: The characteristics of joint pain are widely variable even in patients with similar radiological features. Extra-articular sources are not negligible especially in older patients with a long history of diffuse pain. Differences in pain characteristics among knee areas should be taken into account when examining the pain source.
\end{abstract}

Keywords: Knee; Osteoarthritis; Pain

\section{Introduction}

Knee osteoarthritis (OA) is a major public health problem across the world. Population based studies revealed that symptomatic knee OA is present in $20-30 \%$ of the elderly population aged $>65$ years (Mannoni et al. 2003; Andrianakos et al. 2006) and its prevalence is increasing due in part to the aging of the population (Leveille 2004). Clinical symptoms are dominated by chronic knee joint pain, which leads to disability, psychological distress, and impaired quality of life. Recently, patient-oriented outcome measures have been acquiring greater importance in treating knee OA.

Despite significant advances in the understanding of the pathology of knee OA, pain pathology associated with knee OA remains largely unknown (Dieppe \& Lohmander 2005).

\footnotetext{
* Correspondence: ikeuchim@kochi-u.ac.jp

Department of Orthopaedic Surgery, Kochi Medical School, Kochi University, Kohasu, Oko-cho, Nankoku, Kochi 783-8505, Japan
}

Recently association between symptom and advanced imaging such as MRI (Hunter et al. 2011) and bone scintigraphy (Kraus et al. 2009) is reported. All innervated tissues inside and around the knee joint are potential pain generators in knee OA. In addition, nerves itself, not only peripheral nerves but also central nervous system, plays a significant role in pain mechanisms in knee OA (Lee et al. 2011). Pain generators may differ widely even among patients with similar radiological features, which can contribute to variability in treatment response. It was hypothesized that there would be considerable variation of pain characteristics among patients with similar radiological features. Herein, we examined the main pain sources of medial compartment OA, which is the most common form of knee OA, utilizing a diagnostic joint block, in particular reference to intra-articular tissues. Our study was designed with two purposes: 1) to characterize patients with knee pain predominantly originating from intra-articular structures and 2) to describe the location and pattern of their pain. 


\section{Materials and methods}

A prospective study on patients with knee pain associated with medial OA presenting to a rural medical college hospital was conducted over a 1 year interval (August 2008 - July 2009). Inclusion criteria were American College of Rheumatism clinical criteria (Altman et al. 1986), 100-mm pain visual analogue scale (VAS) greater than $30 \mathrm{~mm}$, radiological OA predominantly localized to medial tibiofemoral joint with grade $\geqq 2$ according to the Kellgren-Lawrence grading system $(0=$ none, $1=$ doubtful, $2=$ minimal, $3=$ moderate and $4=$ severe) (Kellgren \& Lawrence 1957). The pain VAS was used to assess the pain intensity in the knee joint. Patients were asked to rate their knee pain for the last week by placing a mark on a $100 \mathrm{~mm}$ line with two-endpoints representing "no pain (0)" and "worst pain imaginable (100)". The distance along the line from the "no pain" marker was then measured with a ruler giving a pain score out of 100. Pain VAS greater than $30 \mathrm{~mm}$ means moderate to severe knee pain. Exclusion criteria were radiological OA involving lateral tibiofemoral joint or patellofemoral joint with grade $\geqq 2$, inability to walk $200 \mathrm{~m}$ within 5 minutes, mental handicap or psychiatric condition precluding adequate communication, coagulation disturbances, allergies to local anesthetics, previous knee surgeries, and pain originating from hip and spine pathology. Physical examination on hip joints and spine were routinely performed during initial pain assessment. In particular, radiating pain and referred pain associated with motion of hip and spine were examined and excluded in this study. Japanese Orthopaedic Association (JOA) knee score (Wakabayashi et al. 2002), ranging from 0 (worst) to 100 (best), was used to evaluate the clinical state. It consists of four domains: pain on walking (0-30 points), pain on ascending or descending stairs ( $0-25$ points), range of motion (0-35 points), and joint effusion ( $0-10$ points) (Okuda et al. 2012).

The experimental protocol is summarized in Figure 1. Before the initial assessment of knee pain, patients were instructed to walk $200 \mathrm{~m}$ in a hallway at a comfortable speed within 5 minutes. Immediately after the walk, patients were asked to draw a line with pen on their own knees to show the painful area. Then an examiner copied the painful area on a knee pain map developed by (Hill et al. 2003). There are 12 areas with grid lines in this knee pain map (representative anatomical structures within the area); 1 anterior supero-medial (vastus medialis), 2 anterior superior (quadriceps tendon), 3 anterior supero-lateral (iliotibial band and vastus lateralis), 4 anterior medial (medial joint line and medial collateral ligament), 5 patella, 6 anterior lateral (lateral joint line and lateral collateral ligament), 7 patella tendon, 8 pes anserinus, 9 tibial tuberosity, 10 anterior infero-lateral (fibula head and tibialis anterior), 11 anterior infero- medial (tibial insertion of the superficial medial collateral ligament), 12 popliteal (Figure 2). The summation of painful areas was recorded as total painful area. Knee pain during passive extension and flexion was also assessed using the same knee pain map. Examiners (MI and MI) held the patient's leg and gently moved the knee joint to the end of range without over-pressure. Finally, tender points in each area were assessed manually with the patient lying down with knees straight. Examiners applied pressure using the thumb pad to the approximate center of each area for 3 seconds. The pressure force was equivalent to $4 \mathrm{~kg}$, which was sufficient to blanch the nail bed. For a tender point to be considered 'positive', the patient must state that the palpation was painful. If the patient complained of pain in both knees, the more severely affected side was evaluated.

Standard superolateral approach (Zuber 2002) was used for intra-articular injection. A 20G needle was inserted from superolateral side in a sterile manner, and introduced into the suprapatellar pouch, which is a large continuation of the synovial membrane of the knee joint proximal to the trochlea (Dragoo \& Abnousi 2008). Following joint fluid aspiration, $5 \mathrm{ml}$ of $1 \%$ lidocaine were injected into the knee joint, and then $2.5 \mathrm{ml}$ hyaluronic acid (Artz, Kaken seiyaku, Japan) were injected using a same needle. Because previous in vitro studies have shown that local anesthetics are chondrotoxic to human articular cartilage (Grishko et al. 2010; Piper et al. 2011), hyaluronic acid was added in expectation of protecting articular cartilage (Hester et al. 2012; Liu et al. 2012). The knee joint was passively moved from extension to flexion 5 times. Ten minutes after the injection, the patients were instructed to walk $200 \mathrm{~m}$ at a comfortable speed, and then pain assessment was performed as the same as initial assessment. According to previous reports using stringent pain relief criteria (Lovely \& Rastogi 1997; Broadhurst \& Bond 1998; Yeom et al. 2008), a minimum reduction of $70 \%$ in the VAS rating during walk was required to be considered a positive response. A negative response was defined as reduction less than $50 \%$, which is the most commonly used cutoff in the current literature regarding diagnostic blocks (Schwarzer et al. 1994; Liliang et al. 2009; Rupert et al. 2009; Choi et al. 2011). The changes in the area of pain following injection were not evaluated because they were unremarkable. All procedures and assessments were performed by two experienced knee surgeons (MI and MI).

Ethics committee approval from our institution was obtained prior to the study. Before study inclusion, each patient was informed of the objectives and risks of the study and gave his or her consent. The study was conducted in compliance with the Declaration of Helsinki.

In this study, we expediently defined "intra-articular tissues" as joint structures which can be infiltrated with 


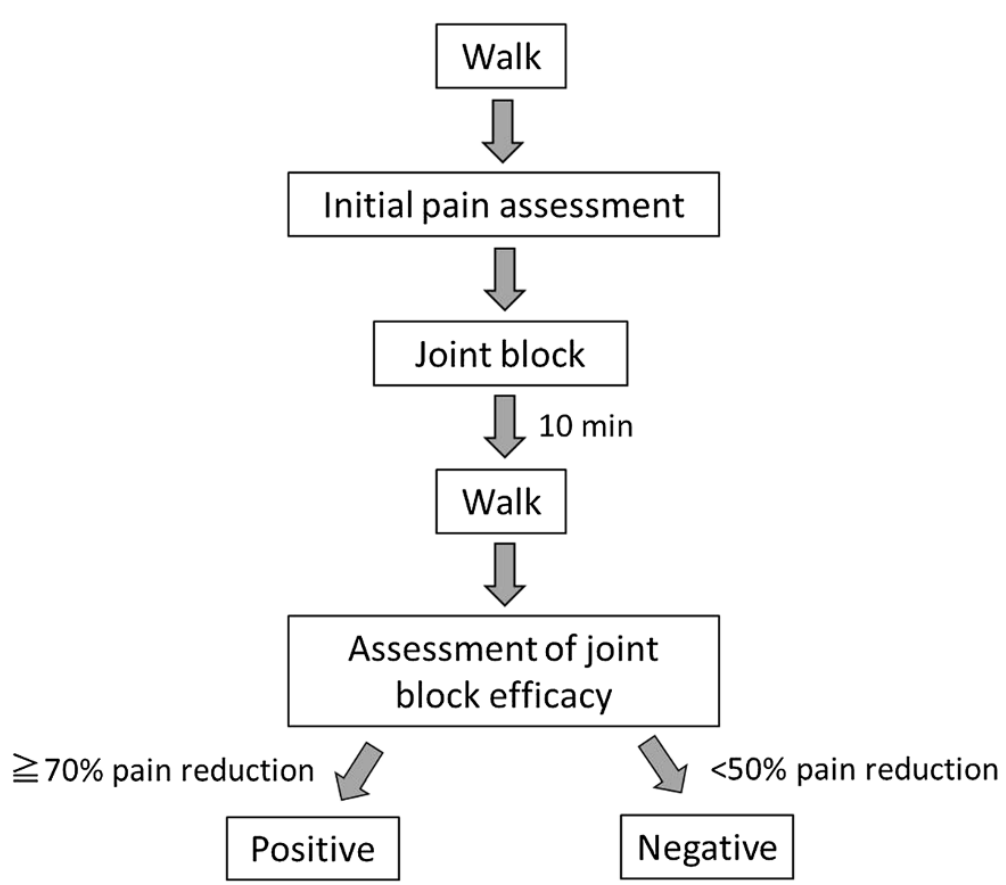

Figure 1 Experimental protocol.

$5 \mathrm{ml}$ of local anaesthetics with $2.5 \mathrm{ml}$ hyaluronic acid, including synovium, cruciate ligaments, exposed subchondral bone and cartilage.

Statistical analyses were performed with the SPSS statistical package for Windows. As this was an observational study, we did not conduct any power analysis for sample size estimation. Mann-Whitney test was used to compare the background characteristics except Kellgren-Lawrence grade 4 (Chi-square test) between joint block-positive and negative patients. $P<0.05$ was regarded as statistically significant.

\section{Results}

One hundred-three patients with medial knee OA were included in this study. There were 85 women and 18 med with a mean age of 72 years (52 to 85). All patients underwent the diagnostic joint block and main sources of knee pain were examined. Sixty-three patients (61\%) had positive response to the joint block, while thirty-three patients (32\%) were regarded as negative. Seven patients who had a $50-70 \%$ pain reduction after joint block were excluded from the analysis. Table 1 shows the result of comparison of baseline characteristics between joint block-positive and negative patients. Patients with negative response were significantly higher age $(p=0.016)$, suffered knee pain for longer time $(p=0.024)$, and complained more diffuse pain $(p=0.003)$. Neither radiological severity nor clinical score were associated with block effects (Table 1).
Among patients in the positive group, the highest prevalence $(82 \%)$ of pain during walk was observed at anterior medial area (area 4), followed by pes anserinus area (area 8) (32\%) and anterior lateral area (area 6) $(30 \%)$ (Figure 3). In addition, pain during walk was sometimes referred to thigh (3 patients) and lower leg (10 patients). Although the highest prevalence (58\%) of pain during extension-flexion of the knee was also observed at anterior medial area (area 4), the next highest prevalence (45\%) was popliteal area (area12) (Figure 3). Specifically, popliteal pain was elicited during flexion in 18 patients, extension in 7 patients and both extension and flexion in 3 patients. Tender points were predominantly localized near the joint line (area 4,6) and pes anserinus (area 8) (Figure 3).

\section{Discussion}

Our results showed that intra-articular tissues were significant source of pain for $61 \%$ patients with medial knee OA. However, older patients with a long history of diffuse pain tended to have negative response to the joint block, indicating the contribution of intra-articular tissues to knee pain was relatively small. One possible explanation for this result is subsequent extra-articular pathology such as decreased elasticity of the soft tissues (Fishkin et al. 2002), neo-innervation of the degenerated tissues (Lian et al. 2006), and central sensitization (Finan et al. 2013). In order to achieve effective pain management for those 


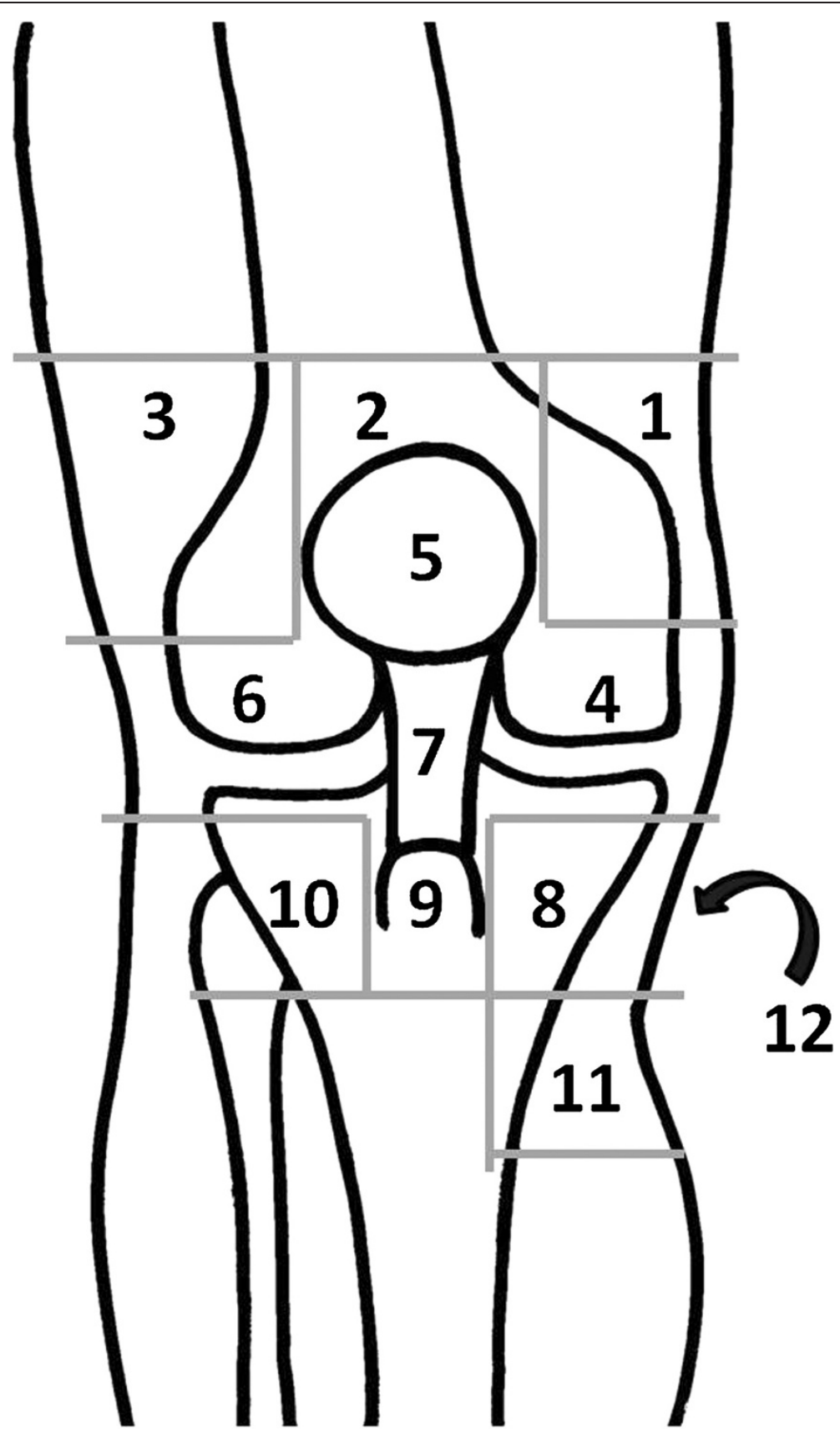

Figure $\mathbf{2}$ Knee pain map. Representative anatomical structures within each numbered area are described in main text in details.

patients, not only intra- but also extra-articular pathology should be targeted. Pain management targeting extraarticular pathology include physical therapy for muscle and soft tissues, drugs such as muscle relaxant and centrally acting analgesics, and cognitive behavioral therapy.

Pain in knee OA involves the peripheral and central nervous system (Lee et al. 2011). Inflammatory mediators inside the joint sensitize nociceptors innervating synovium, leading to local areas of enhanced pain sensitivity. Eventually, growth of new nociceptors and activation of nociceptors in the exposed subchondral bone augment the peripheral input to the central nervous system. Central pain resulted from central sensitization and reduced central inhibition leads to prolonged and enhanced pain (Sluka et al. 2012). In this study, pain originating from intraarticular structures in medial knee OA was not always localized at synovial cavity of the joint, let alone medial side. For instance, although there is no synovial cavity in area 8 (around pes anserinus), pain was relieved by joint block in the positive group. If the local insults caused pain 
Table 1 Comparison of baseline characteristics between joint block-positive and negative patients

\begin{tabular}{cccc}
\hline & \multicolumn{2}{c}{ Response to joint block } & P value \\
\cline { 2 - 3 } & Positive & Negative & \\
\hline Age (yrs) & $70(10)$ & $76(6)$ & $0.016^{*}$ \\
Disease duration (mo) & $72[12,180]$ & $120[50,240]$ & $0.024^{*}$ \\
JOA knee score (pts) & $63(14)$ & $58(15)$ & 0.090 \\
Pain BAS during walk (mm) & $64(19)$ & $62(15)$ & 0.653 \\
Kellgren-lawrence grade 4 (\%)† & 48 & 48 & 0.995 \\
Knee range of motion (deg) & $122(22)$ & $125(17)$ & 0.708 \\
Total painful area & $2[1,4]$ & $4[3,7]$ & $0.003^{* *}$ \\
\hline
\end{tabular}

Values are mean (standard deviation) and median [inter-quartile range].

Mann-Whitney test was used except + Chi-square test. ${ }^{*} p<0.05$ and ${ }^{* *} p<0.01$.

in area 8 , joint block was theoretically not effective. Therefore, we think that the pain mechanism of diffuse pain is at least partly due to alterations in central pain processing.

Pain characteristics differed among different knee areas. For instance, pain at anterior medial area (area 4) was the most common finding in patients with medial knee OA. This pain was observed during walk rather than during extension-flexion, which might reflect increased load at medial compartment in genu varum. On the contrary, popliteal pain (area 12) was observed during extensionflexion of the knee rather than during walk, which might reflect impingement of torn meniscus and capsular contracture. In addition, pain at anterior medial area was usually accompanied by tenderness, while popliteal pain was not. It is possible that the presence of tenderness only reflects the superficial location of painful structures.
However, knowing the pain characteristics at each knee area is helpful in evaluating pain in knee OA during daily practice.

Several authors used diagnostic blocks with a criterion of $50 \%$ pain relief for knee joint (Choi et al. 2011), sacroiliac joint (Rupert et al. 2009), lumbar facet joint (Schwarzer et al. 1994), and shoulder joint (Liliang et al. 2009). In this study, a more stringent criterion, $70 \%$ pain relief according to (Broadhurst \& Bond 1998), was used. A response of $70 \%$ pain relief could be inferred to indicate that the intra-articular tissues were not sole but significant source of pain. Although $70 \%$ criteria were to decrease the false-positive rate, it might have resulted in increased false-negative cases vice versa. It seemed more problematic to include the false-positive cases because the main purpose of this study was not to clarify the prevalence of pain originated from intra-articular tissues but to describe its characteristics.

The strength of this study is detailed pain assessment using joint block. However, there are several limitations in our study. Firstly, a placebo response might have been involved because of our single uncontrolled block. For an ideal prevalence study, a placebo controlled or comparative local anaesthetic study will be necessary. Secondly, intraarticular injection was performed blindly. Although the superolateral approach is reported as the most accurate approach for intra-articular knee injection (Hermans et al. 2011), ultrasound guidance (Berkoff et al. 2012) might have resulted in the better accuracy of injection. Thirdly, spread of local anaesthetics could be individually variable. Future clinical trials using a double-injection paradigm for patient selection and a control group are

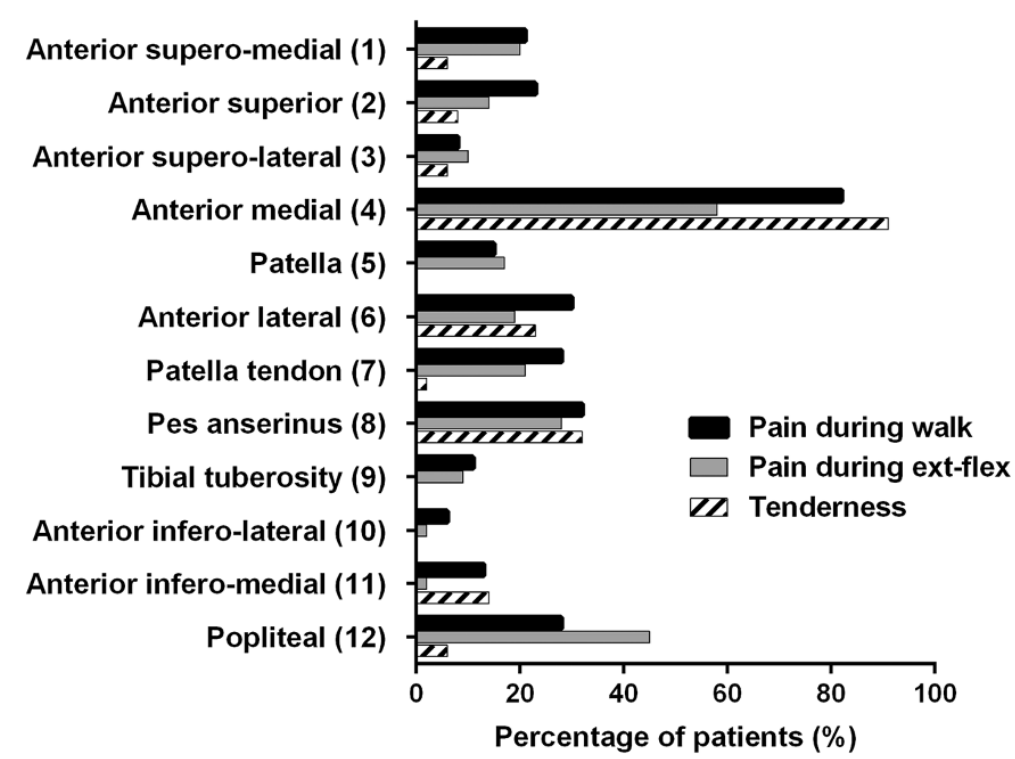

Figure 3 Percentage of patients with pain and tenderness in each area. The number in parentheses is equal to the number in the knee pain map (Figure 2). ex: extension-flexion. 
needed to further clarify the pain referral patterns observed in knee OA. Lastly, the problems with the study design include observational study with relatively small sample size. Despite these limitations, we believe that this study provides clinically important information on pain characteristics associated with medial compartment knee OA.

In conclusion, our results suggest that the characteristics of joint pain are widely variable even in patients with similar radiological features. Although the main source of pain in medial osteoarthritis knees is intra-articular structures, extra-articular sources are not negligible especially in older patients with a long history of diffuse pain. Differences in pain characteristics among knee areas should be taken into account when examining the pain source of knee osteoarthritis.

\section{Competing interests}

No benefits in any form have been received or will be received from a commercial party related directly or indirectly to the subject of this article.

\section{Authors' contributions}

$\mathrm{Ml}$ and $\mathrm{MI}$ designed the study, analyzed and interpreted the results, and wrote the manuscript. KA and NS analyzed and interpreted the results. TT directed the study. All authors read and approved the final manuscript.

Received: 26 July 2013 Accepted: 18 November 2013

Published: 23 November 2013

\section{References}

Altman R, Asch E, Bloch D, Bole G, Borenstein D, Brandt K, Christy W, Cooke TD, Greenwald R, Hochberg M et al (1986) Development of criteria for the classification and reporting of osteoarthritis: classification of osteoarthritis of the knee: diagnostic and therapeutic criteria committee of the American rheumatism association. Arthritis Rheum 29:1039-1049

Andrianakos AA, Kontelis LK, Karamitsos DG, Aslanidis SI, Georgountzos Al, Kaziolas GO, Pantelidou KV, Vafiadou EV, Dantis PC (2006) Prevalence of symptomatic knee, hand, and hip osteoarthritis in Greece: The ESORDIG study. J Rheumatol 33:2507-2513

Berkoff DJ, Miller LE, Block JE (2012) Clinical utility of ultrasound guidance for intra-articular knee injections: a review. Clin Interv Aging 7:89-95

Broadhurst NA, Bond MJ (1998) Pain provocation tests for the assessment of sacroiliac joint dysfunction. J Spinal Disord 11:341-345

Choi WJ, Hwang SJ, Song JG, Leem JG, Kang YU, Park PH, Shin JW (2011) Radiofrequency treatment relieves chronic knee osteoarthritis pain: a double-blind randomized controlled trial. Pain 152:481-487

Dieppe PA, Lohmander LS (2005) Pathogenesis and management of pain in osteoarthritis. Lancet 365:965-973

Dragoo JL, Abnousi F (2008) Disorders of the suprapatellar pouch of the knee. Knee 15:348-354

Finan PH, Buenaver LF, Bounds SC, Hussain S, Park RJ, Haque UJ, Campbell CM, Haythornthwaite JA, Edwards RR, Smith MT (2013) Discordance between pain and radiographic severity in knee osteoarthritis: findings from quantitative sensory testing of central sensitization. Arthritis Rheum 65:363-372

Fishkin Z, Miller D, Ritter C, Ziv I (2002) Changes in human knee ligament stiffness secondary to osteoarthritis. J Orthop Res 20:204-207

Grishko V, Xu M, Wilson G, Pearsall AW (2010) Apoptosis and mitochondria dysfunction in human chondrocytes following exposure to lidocaine, bupivacaine, and ropivacaine. J Bone Joint Surg Am 92:609-618

Hermans J, Bierma-Zeinstra SM, Bos PK, Verhaar JA, Reijman M (2011) The most accurate approach for intra-articular needle placement in the knee joint: a systematic review. Semin Arthritis Rheum 41:106-115

Hester W, Yang J, Wang GY, Liu S, O'Brien MJ, Savoie FH, You Z (2012) Hyaluronan does not affect bupivacaine's inhibitory action on voltage-gated potassium channel activities in bovine articular chondrocytes. Adv Orthop 2012:361534
Hill CL, Gale DR, Chaisson CE, Skinner K, Kazis L, Gale ME, Felson DT (2003) Periarticular lesions detected on magnetic resonance imaging: prevalence in knees with and without symptoms. Arthritis Rheum 48:2836-2844

Hunter DJ, Zhang W, Conaghan PG, Hirko K, Menashe L, Li L, Reichmann WM Losina $E$ (2011) Systematic review of the concurrent and predictive validity of MRI biomarkers in OA. Osteoarthritis Cartilage 19:557-588

Kellgren JH, Lawrence JS (1957) Radiological assessment of osteo-arthrosis. Ann Rheum Dis 16:494-502

Kraus VB, McDaniel G, Worrell TW, Feng S, Vail TP, Varju G, Coleman RE (2009) Association of bone scintigraphic abnormalities with knee malalignment and pain. Ann Rheum Dis 68:1673-1679

Lee YC, Nassikas NJ, Clauw DJ (2011) The role of the central nervous system in the generation and maintenance of chronic pain in rheumatoid arthritis, osteoarthritis and fibromyalgia. Arthritis Res Ther 13:211

Leveille SG (2004) Musculoskeletal aging. Curr Opin Rheumatol 16:114-118

Lian O, Dahl J, Ackermann PW, Frihagen F, Engebretsen L, Bahr R (2006) Pronociceptive and antinociceptive neuromediators in patellar tendinopathy. Am J Sports Med 34:1801-1808

Liliang PC, Lu K, Liang CL, Tsai YD, Hsieh CH, Chen HJ (2009) Pulsed radiofrequency lesioning of the suprascapular nerve for chronic shoulder pain: a preliminary report. Pain Med 10:70-75

Liu S, Zhang QS, Hester W, O'Brien MJ, Savoie FH, You Z (2012) Hyaluronan protects bovine articular chondrocytes against cell death induced by bupivacaine at supraphysiologic temperatures. Am J Sports Med 40:1375-1383

Lovely TJ, Rastogi P (1997) The value of provocative facet blocking as a predictor of success in lumbar spine fusion. J Spinal Disord 10:512-517

Mannoni A, Briganti MP, Di Bari M, Ferrucci L, Costanzo S, Serni U, Masotti G, Marchionni N (2003) Epidemiological profile of symptomatic osteoarthritis in older adults: a population based study in Dicomano, Italy. Ann Rheum Dis 62:576-578

Okuda M, Omokawa S, Okahashi K, Akahane M, Tanaka Y (2012) Validity and reliability of the Japanese orthopaedic association score for osteoarthritic knees. J Orthop Sci 17:750-756

Piper SL, Kramer JD, Kim HT, Feeley BT (2011) Effects of local anesthetics on articular cartilage. Am J Sports Med 39:2245-2253

Rupert MP, Lee M, Manchikanti L, Datta S, Cohen SP (2009) Evaluation of sacroiliac joint interventions: a systematic appraisal of the literature. Pain Physician 12:399-418

Schwarzer AC, Aprill CN, Derby R, Fortin J, Kine G, Bogduk N (1994) Clinical features of patients with pain stemming from the lumbar zygapophysial joints: is the lumbar facet syndrome a clinical entity? Spine (Phila Pa 1976) 19:1132-1137

Sluka KA, Berkley KJ, O'Connor MI, Nicolella DP, Enoka RM, Boyan BD, Hart DA, Resnick E, Kwoh CK, Tosi LL, Coutts RD, Kohrt WM (2012) Neural and psychosocial contributions to sex differences in knee osteoarthritic pain Biol Sex Differ 3:26

Wakabayashi S, Akizuki S, Takizawa T, Yasukawa Y (2002) A comparison of the healing potential of fibrillated cartilage versus eburnated bone in osteoarthritic knees after high tibial osteotomy: An arthroscopic study with 1-year follow-up. Arthroscopy 18:272-278

Yeom JS, Lee JW, Park KW, Chang BS, Lee CK, Buchowski JM, Riew KD (2008) Value of diagnostic lumbar selective nerve root block: a prospective controlled study. AJNR Am J Neuroradiol 29:1017-1023

Zuber TJ (2002) Knee joint aspiration and injection. Am Fam Physician 66:1497-1500, 1503-1494, 1507

\section{doi:10.1186/2193-1801-2-628}

Cite this article as: Ikeuchi et al.: Clinical characteristics of pain originating from intra-articular structures of the knee joint in patients with medial knee osteoarthritis. SpringerPlus 2013 2:628. 